\title{
Brain Extracellular Quinolinic Acid in Chronic Experimental Hepatic Encephalopathy as Assessed by In Vivo Microdialysis: Acute Effects of L-Tryptophan
}

Peter B. F. Bergqvist, B.Sc., Melvyn P. Heyes, Ph.D., Gustav Apelquist, M.D., Roger F. Butterworth, Ph.D., and Finn Bengtsson, M.D., Ph.D.

Increased brain quinolinic acid (QUIN) levels have been suggested to play a role in hepatic encephalopathy (HE). Previous brain tissue studies have been unable to confirm this hypothesis. Because QUIN is a potent NMDA-receptor agonist, it also is relevant to determine brain extracellular QUIN levels in HE. For this purpose, we assessed frontal neocortical extracellular QUIN levels by in vivo microdialysis in rats subjected to a portacaval shunt (PCS). We also evaluated the acute effects of altered L-tryptophan ( $L-T R P)$ availability on brain extracellular QUIN levels.
The basal extracellular L-TRP levels were significantly ( $p<$ $.001)$ higher in the PCS rats than in the sham-operated controls. However, the QUIN level $(p<.05)$ and the QUIN to L-TRP ratio $(p<.01)$ were significantly lower in the $P C S$ rats. Elevated L-TRP availability increased the QUIN levels to a similar degree in both sham and PCS rats. This study, in conjunction with our previous results, does thereby not support a major involvement of QUIN in the pathogenesis of HE. [Neuropsychopharmacology 15:382-389, 1996]
KEY WORDS: Hepatic encephalopathy; In Vivo cerebral microdialysis; Kynurenines; Liver disease; Portacaval anastomosis; Quinolinic acid

The pathogenesis of the neuropsychiatric syndrome associated with a variety of both acute and chronic liver diseases, better known as hepatic encephalopathy (HE), is still unknown. In patients with varying degrees of HE, the cerebrospinal fluid (Ono et al. 1978; Smith et al.

From the Department of Clinical Pharmacology (PBFB, GA, FB), Lund University, Lund, Sweden; the Section on Analytical Biochemistry (MPH), Laboratory of Clinical Science, National Institute of Mental Health, Bethesda, MD; and the Neuroscience Research Unit (RFB), Saint-Luc Hospital, University of Montreal, Montreal, Quebec, Canada.

Address correspondence to: Finn Bengtsson, M.D., Ph.D., Department of Clinical Pharmacology, Lund University Hospital, S-221 85 Lund, Sweden, Tel. +46-46-17 33 51, Fax +46-46-211 1987.

Received August 17, 1995; revised October 31, 1995; accepted November 9, 1995.
1978; Hutson et al. 1979; Cascino et al. 1982; Rössle et al. 1984), as well as postmortem brain tissue (Jellinger et al. 1978; Bergeron et al. 1989), levels of the aromatic amino acid L-tryptophan (L-TRP) have convincingly been shown to be increased. Elevated L-TRP levels have also been clearly demonstrated in brain tissue (Baldessarini and Fischer 1979; Curzon et al. 1975; Cummings et al. 1976; Mans et al. 1982, 1990; Bengtsson et al. 1991) and in the brain extracellular fluid (Bergqvist et al. 1995b) in portacaval shunted (PCS) rats, a commonly used and nontoxin-induced experimental in vivo model of chronic $\mathrm{HE}$. L-TRP is an essential component in all vertebrate diets, and the pharmacological effects often seen directly as the results of L-TRP augmentation or depletion of the food indicate a central role for cell-to-cell communication promoted by this amino acid. Furthermore, L-TRP is the precursor of many neuroactive compounds such as serotonin, 5-hydroxytryptophol, melatonin, and tryptamine, as well as for compounds metabolized via the kynuren- 
ine pathway. Among the kynurenine pathway metabolites, quinolinic acid (QUIN; pyridine-2,3-dicarboxylic acid) seems to be of particular pharmacological and functional importance (for an overview, see Stone 1993). QUIN is an endogenous agonist of the NMDA-receptor, mediating neuronal membrane calcium ion transients and thereby excitatory neurotransmission in the brain (Stone 1993).

In the mid-1980s elevated brain QUIN levels were reported in humans with HE as well as in PCS and acute hyperammonemic rats (Moroni et al. 1986a, 1986b). In a recent study, however, decreased rather than increased plasma and brain tissue QUIN levels were found in PCS rats (Bergqvist et al. 1995a). Furthermore, no parallel change between neurological symptoms in acute hyperammonemia with brain QUIN levels occurred (Bergqvist et al. 1995a). Because QUIN is a potent excitatory receptor ligand in vivo, there is still a possibility that relatively minor elevations of QUIN in the extracellular environment in the brain could play a role in the pathogenesis of HE (Bengtsson 1992; Butterworth 1992).

In the present study, we determined the frontal neocortical extracellular L-TRP and QUIN levels in chronic PCS rats using the in vivo microdialysis technique. First, we determined basal extracellular levels of L-TRP and QUIN 5 to 6 weeks following permanent surgical PCS. Second, we also assessed the acute effects of systemic pharmacological manipulation of L-TRP availability on the extracellular levels of QUIN at this point.

\section{MATERIALS AND METHODS}

\section{Animals and Surgery}

Male Sprague-Dawley rats weighing 175 to $200 \mathrm{~g}$ (Möllegaard Breeding \& Research Centre A/S, Skensved, Denmark) were used. The animals had free access to tap water and standard laboratory pelleted chow containing $21 \%$ crude protein (product R3; Ewos AB, Södertälje, Sweden). No pair feeding of controls was conducted. The rats were housed in groups of three animals in standard macrolone cages with sawdust bedding under climate-controlled conditions for temperature and humidity. The rats were kept in a 12-hour light/dark cycle synchronous with daylight (lights on at 6.00 A.M.). The study was approved by the Animal Ethics Committee at Lund University.

A permanent surgical end-to-side PCS was created with some modifications (Bengtsson et al. 1991; Bergqvist et al. 1995a). The control rats underwent sham operation (sham) identical to the PCS procedure except for the performance of the portacaval anastomosis. The PCS and the sham rats were allowed to recuperate for 5 to 6 weeks after intraabdominal surgery before the microdialysis experiments were performed. The rats were controlled for body weight at time of the PCS-procedure, thereafter weekly and at termination of the experiments. At time of sacrifice the liver weight was recorded, and the liver-to-body weight ratio was calculated.

\section{In Vivo Microdialysis}

The microdialysis probes were prepared as described by Kalén et al. (1988), using GFE Plus16 (Gambro, Lund, Sweden) hemophane tubing (OD $0.22 \mathrm{~mm}$ and ID 0.20 $\mathrm{mm}$ ) with a molecular cutoff of $7 \mathrm{kDa}$. The probes were implanted into the frontal neocortex (coordinates relative to bregma: rostral, $+3.2 \mathrm{~mm}$; lateral, $-1.5 \mathrm{~mm}$; ventral, $-2.5 \mathrm{~mm}$; Paxinos and Watson 1982) under halothane anaesthesia (ISC Chemicals Ltd., Avonmouth, England) the day prior to the dialysis procedure. The subsequent day, the probe inlet was attached to a liquid swivel that was connected to a microinfusion system (model CMA/ 100; Bioanalytical Systems Inc. [BAS], West Lafayette, IN). All microdialysis experiments were conducted on conscious and freely moving animals. The probes were perfused at $2.5 \mu \mathrm{L} /$ minute with an isotonic Ringer's buffer (Pharmacia AB, Uppsala, Sweden; NaCl 147 $\mathrm{mmol} / \mathrm{L}, \mathrm{KCl} 4 \mathrm{mmol} / \mathrm{L}, \mathrm{CaCl}_{2} 2.4 \mathrm{mmol} / \mathrm{L}, \mathrm{pH}$ 6.0). No samples were collected during an initial stabilization period of 45 minutes. Then, samples were collected in 15-minute fractions to obtain an adequate baseline. The samples were immediately frozen in liquid nitrogen without addition of any antioxidants and stored at $-70^{\circ} \mathrm{C}$ until the subsequent neurochemical analyses were performed. Not all dialysis samples collected were analyzed for QUIN or L-TRP, but they were used for other kinds of chemical analyses and included in other investigations.

After collection of the baseline fractions, the L-TRP availability was manipulated pharmacologically by systemic administration of a single loading dose of L-TRP ( 280 mg/kg I.P.; Sigma Chemical Co., St. Louis, MO) dissolved in isotonic saline. The first L-TRP injection was repeated 3 hours later by four injections of lower doses of L-TRP (50 mg/ $\mathrm{kg}$ each) during the four consecutive hours to follow. The total volume of fluid administered this way during the 6.5-hours experiment was about 2 $\mathrm{mL} / 100 \mathrm{~g}$ of body weight. In a number of animals from each group (sham and PCS) L-TRP was replaced by an equimolar amount of saline (Sigma) to account for variables such as possible nonspecific peritoneal irritation and osmotic effects. Thus, four experimental groups denoted as sham-saline $(n=5)$, sham-L-TRP $(n=4)$, PCSsaline $(n=4)$, and PCS-L-TRP $(n=4)$ were investigated.

Diurnal fluctuations in the concentration of brain large neutral amino acids (e.g., L-TRP) are known to exist (Crandall and Fernstrom 1983). Therefore, we also 
assessed the basal levels of L-TRP 24 hours after initiating the microdialysis procedure.

\section{Neurochemistry}

L-Tryptophan. The dialysates content of L-TRP was determined by a method modified from Hjorth and Sharp (1993) applying a high-performance liquid chromatography (HPLC) assay with electrochemical detection. In brief, the samples were separated at $30^{\circ} \mathrm{C}$ on a $2.0-\mathrm{mm} \times 15-\mathrm{cm}$ stainless steel column packed with Ultrasphere ODS $5-\mu \mathrm{m}$ material (Beckman Instruments Inc., Fullerton, $\mathrm{CA}$ ). The mobile phase consisted of $0.126 \mathrm{M} \mathrm{NaH}_{2} \mathrm{PO}_{4}$ (pH 4.0) containing $0.85 \mathrm{mM}$ EDTA, $0.03 \mathrm{mM}$ sodium n-octyl sulfate, and $10 \%$ (vol/vol) HPLC-grade methanol. An aliquot of $20 \mu \mathrm{L}$ of dialysate was manually injected with a Rheodyne injector (model 7125; Rheodyne Inc., Cotati, CA). The mobile phase was delivered at $0.25 \mathrm{~mL} /$ minute through a twin-reciprocating pump (LKB-2150; LKB-Produkter AB, Bromma, Sweden). The L-TRP was detected by a glassy carbon working electrode set at $+0.80 \mathrm{~V}$ relative to an $\mathrm{Ag} / \mathrm{AgCl}$ reference electrode (LC-4B; BAS), and the detection signals were recorded on an integrator (SP 4290; SpectraPhysics, San Jose, CA). The detection limit (signal-tonoise ratio, $3: 1$ ) for L-TRP was 0.1 pmol.

Quinolinic acid. The QUIN content of the dialysate samples was determined according to a method previously described in great detail (Heyes and Markey 1988a, $1988 \mathrm{~b}$ ) using $\left[{ }^{18} \mathrm{O}\right]$ QUIN as internal standard (see also Bergqvist et al. 1995a). In short, after derivatization with hexafluoroisopropanol (HFIP) the samples were analyzed with a gas chromatograph $=$ mass spectrometer (GC = MS; Hewlett-Packard 5988A quadrapole mass filter) operating in an electron-capture negative chemical ionization mode using volatile methane and helium as reagent and carrier gases, respectively. The QUINHFIP and $\left[{ }^{18} \mathrm{O}\right] \mathrm{QUIN}-\mathrm{HFIP}$ derivatives were quantified by peak areas of the molecular anions [mass charge ratio $(\mathrm{m} / \mathrm{z})$ of 467 and 471 , respectively]. The detection limit of this method (signal-to-noise ratio, 25:1) for QUIN was $0.3 \mathrm{fmol}$.

\section{Statistics}

All values are expressed as means \pm SEM. A probability of less than $5 \%(p<.05)$ was preset to signify significant differences between groups. All statistical analyses were performed by using the computer software StatView 4.0 (Abacus Concepts, Inc., Berkeley, CA). When two groups were compared, a two-tailed Student's $t$-test was used. When more than two groups were compared, a one-factor analysis of variance (ANOVA) was used. If significance was reached with the ANOVA, a post hoc analysis (Fis- cher's PLSD-test) was applied to determine which groups differed.

\section{RESULTS}

\section{Weight Measurements}

The administration of L-TRP was shown not to affect the body and liver weight parameters in either the PCS or sham groups. Hence, the data from these measurements were pooled into two groups representing only sham or PCS. At termination of the experiment 5 to 6 weeks postsurgery, there was a significant $(p<.001)$ difference in body weight between sham $(399 \pm 8 \mathrm{~g}, n=$ 9) and PCS rats (273 $\pm 16 \mathrm{~g}, n=8)$. The body weight gain from the time of operation to sacrifice in sham (133 \pm $7 \mathrm{~g})$ and PCS (11 $\pm 8 \mathrm{~g})$ indicated that both groups had reached a postsurgical anabolic state by the time the dialysis experiments were performed. A significant $(p<$ $.001)$ difference in the liver-to-body weight ratio between the sham rats $(3.6 \pm 0.1 \%, n=9)$ and the chronic PCS rats $(2.3 \pm 0.1 \%, n=8)$ was present at sacrifice.

\section{Basal Frontal Neocortical Extracellular L-TRP and QUIN Concentrations}

Five to six weeks following the PCS procedure the extracellular L-TRP levels in the PCS rats were significantly $(p<.001)$ higher than in the sham-operated controls (Table 1). The brain extracellular QUIN levels as well as QUIN-to-L-TRP ratios were, however, significantly lower $(p<.05$ and $p<.01$, respectively) in the $\mathrm{PCS}$ rats than in the sham rats.

\section{Effects of an Exogenous Systemic L-TRP Challenge on the Frontal Neocortical Extracellular L-TRP and QUIN Levels}

The PCS and sham rats exposed to saline injections displayed a continuous and comparable longitudinal lowering of the extracellular L-TRP during the 6.5-hour course of dialysis (circles in Figure 1). This tendency to-

Table 1. Basal Extracellular L-TRP and QUIN Levels $(\mathrm{nmol} / \mathrm{L})$ and QUIN to L-TRP ratio (\%) in PCS and ShamOperated Rats 5 to 6 Weeks Following the PCS Procedure

\begin{tabular}{lcc}
\hline & Sham $(\boldsymbol{n}=\mathbf{9})$ & PCS $(\boldsymbol{n}=\mathbf{8})$ \\
\hline L-TRP & $263 \pm 36$ & $795 \pm 110^{* * *}$ \\
QUIN & $9.41 \pm 0.91$ & $7.00 \pm 0.55^{*}$ \\
QUIN/L-TRP & $20.8 \pm 3.7$ & $4.80 \pm 0.62^{* *}$ \\
\hline
\end{tabular}

All values are means \pm SEM (uncorrected for probe recovery). Values in the PCS group significantly different from the sham group are indicated as

${ }^{*} p<.05$

$* * p<.01$, and

${ }^{* * *} p<.001$ (Student's $t$-test) 


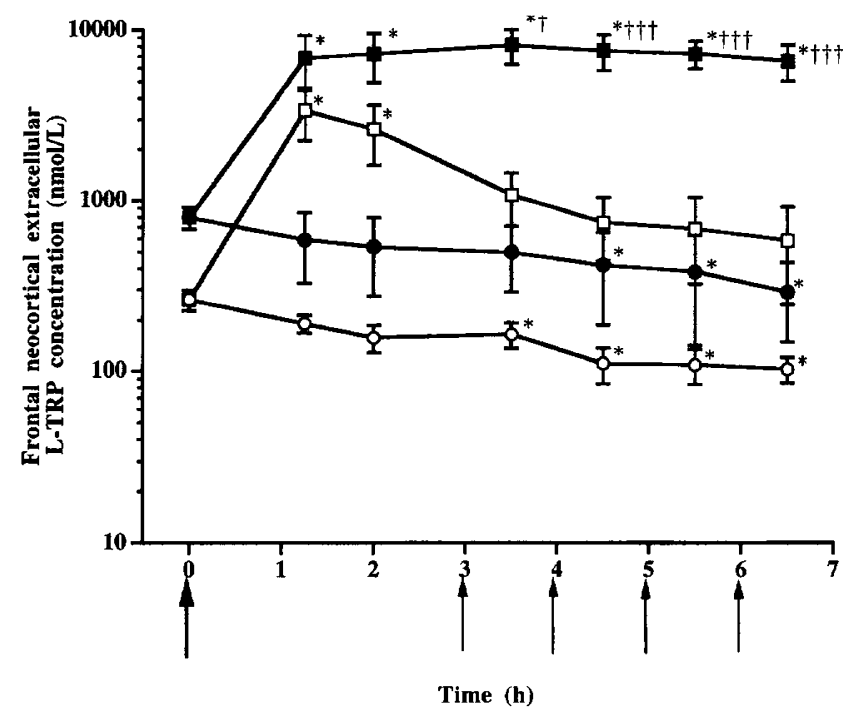

Figure 1. The brain extracellular L-TRP during a 6.5-hour period of continuous neocortical dialysis following challenges of L-TRP or saline to PCS and sham-operated rats. Thick arrow, administration of a single loading dose of L-TRP ( $280 \mathrm{mg} / \mathrm{kg}$ body weight I.P.), thin arrows, four consecutive injections of lower L-TRP doses $(50 \mathrm{mg} / \mathrm{kg}$ I.P.). Open circles, sham-saline; open squares, sham-L-TRP; solid circles, PCSsaline; solid squares, PCS-L-TRP. All values are means \pm SEM and expressed as nanomoles per liter (uncorrected for probe recovery). ${ }^{*}$, Significant $(p<.05)$ difference compared to respective basal value within each group (ANOVA). + and +++ , Significant ( $p<.05$ and 0.001 , respectively) differences between PCS-L-TRP and sham-L-TRP (ANOVA).

ward lower L-TRP levels was proven to be significant from 3.5 hours and onward for sham-saline and from 4.5 hours onward for the PCS-saline rats. However, 24 hours after the start of dialysis these L-TRP values had returned to $415 \pm 270 \mathrm{nmol} / \mathrm{L}$ for sham-saline $(n=3)$ and $1310 \pm 910 \mathrm{nmol} / \mathrm{L}$ for PCS-saline $(n=3)$. These latter values are not shown in Figure 1, but the data were not found to be statistically different from those obtained as basal values before the challenge. In order to obtain these data, the dialysis probe had to be kept in place overnight. Because of some difficulties for dialysis probe patency during this extended period of time, not all animals could present dialysates after 24 hours of a technical quality acceptable for L-TRP analysis, which thereby increased the variability of the results within each group.

As early as 75 minutes after the initial L-TRP challenge, the sham-L-TRP and PCS-L-TRP groups displayed both significantly $(p<.05)$ higher L-TRP levels (approximately 14- and 8-fold) than baseline values for the groups, respectively (squares in Figure 1). The L-TRP level thereafter diminished continuously in the shamL-TRP rats despite the addition of successive lower doses of L-TRP during the 6.5-hour course of dialysis.
Conversely, for the PCS-L-TRP rats the elevated concentrations of L-TRP following the challenges remained at about the same level during the whole 6.5-hour period of dialysis. Accordingly, differences in time course of the extracellular L-TRP between sham and PCS following this procedure of exogenous L-TRP challenge were evident; this is shown in Figure 1 as significantly higher L-TRP levels in the PCS-L-TRP versus sham-L-TRP following 3.5 hours of the challenge $(p<.05)$ and sustained at 4.5, 5.5, and 6.5 hours, respectively $(p<.001)$. At the 24-hour time point the L-TRP levels had returned to $790 \pm 530 \mathrm{nmol} / \mathrm{L}$ for sham-L-TRP $(n=3)$ and 1,040 $\pm 690 \mathrm{nmol} / \mathrm{L}$ for PCS-L-TRP $(n=2)$. None of these values were found to be statistically different from those obtained prior to the L-TRP challenge.

Increases in L-TRP availability led to higher levels of brain extracellular QUIN to a comparable degree in both the sham-L-TRP and PCS-L-TRP groups (squares in Figure 2). The initial loading dose of L-TRP resulted in significantly higher QUIN levels at 2.5 hours and onward following the loading L-TRP challenge than basal value in both sham and PCS rats. The subsequent four successive doses of L-TRP resulted in significantly elevated steady-state QUIN levels as compared to the basal values in all rats subjected to this treatment. These steady-state QUIN levels in the PCS-L-TRP and sham-

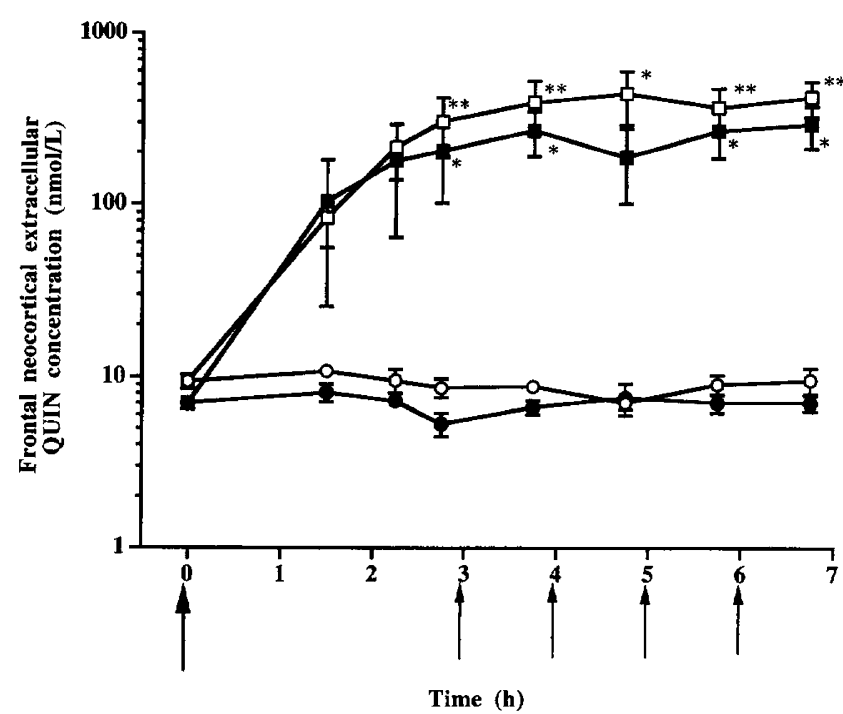

Figure 2. The brain extracellular QUIN levels during a 6.5hour period of continuous neocortical dialysis following challenges of L-TRP or saline to PCS and sham-operated rats. Thick arrow, administration of a single loading dose of L-TRP ( $280 \mathrm{mg} / \mathrm{kg}$ boy weight I.P.); thin arrows, four consecutive injections of lower L-TRP doses (50 mg/ $\mathrm{kg}$ I.P.); open circles, sham-saline; open squares, sham-L-TRP; solid circles, PCS-saline; solid squares, PCS-L-TRP. All values are means \pm SEM and expressed as nanomoles per liter (uncorrected for probe recovery). ${ }^{*}$ and ${ }^{* *}$, Significant $(p<.05$ and .01 , respectively) differences compared to respective basal value within each group (ANOVA). 
L-TRP groups were about 30- and 20-fold higher than the respective basal QUIN values and were both equivalent to QUIN levels of about $200 \mathrm{nmol} / \mathrm{L}$. Although statistically nonsignificant, the area under curve (AUC) values were about $30 \%$ lower for the PCS-L-TRP rats than for the sham-L-TRP animals $(77.1 \pm 7.7 \mu \mathrm{mol} / \mathrm{L} \times$ minute versus $107 \pm 33 \mu \mathrm{mol} / \mathrm{L} \times$ minute), indicating a generally lower accumulation of QUIN following L-TRP administration in the PCS rats. The administration of saline did not significantly affect the QUIN levels either in the sham-saline or in PCS-saline groups.

\section{DISCUSSION}

It has been suggested that the endogenous NMDAreceptor agonist QUIN plays a role in several central nervous system (CNS) diseases, including epilepsy, the neuropathology frequently associated with acquired immunodeficiency syndrome, Huntington's disease, as well as in HE (for an overview, see Stone 1993). In more detail, elevated brain QUIN levels in humans with HE as well as in PCS and acute hyperammonemic rats led to a hypothesis that increased L-TRP availability in the brain may subsequently increase brain QUIN levels and that elevated brain QUIN levels could contribute to the neurological alterations observed in HE (Moroni et al. 1986a, 1986b). More recent data, however, do not support the contention of a major role for QUIN in the pathogenesis of HE (Basile et al. 1995a, 1995b; Bergqvist et al. 1995a). The previous studies assessing brain QUIN in the development of HE have, however, addressed this issue by investigating only brain tissue levels of this potential neurotoxin. Based on the fact that QUIN is a potent competetive NMDA-receptor agonist in vivo the present study was designed to provide necessary complementary information on QUIN levels in the brain extracellular fluid in chronic HE.

The present study reports two novel findings concerning brain QUIN in experimental chronic HE. First, basal frontal neocortical extracellular levels of QUIN at 5 to 6 weeks of chronic PCS were significantly lower than those for sham-operated controls. These decreased (by $26 \%$ ) basal frontal neocortical extracellular fluid QUIN levels are in agreement with the reduced (by $27 \%$ ) basal neocortical tissue QUIN levels also seen in chronic PCS rats (Bergqvist et al. 1995a). Hence, chronic $\mathrm{HE}$ is probably not associated with increases either in neocortical brain tissue or extracellular fluid QUIN concentrations. The second finding relates to the observation of a rather similar accumulation profile for brain extracellular QUIN in both PCS and sham rats following increased availability to L-TRP. The only difference in brain extracellular QUIN observed between sham and PCS rats following pharmacological manipulation of the L-TRP availability was a nonsignificant decrease in the AUC for the QUIN accumulation in the PCS rats compared with the sham-operated controls. The parallelism between PCS and sham-operated rats is rather unexpected in view of previous reports of significant alterations of L-TRP metabolism following hepatic failure in human (Rössle et al. 1986) and rats (Bengtsson et al. 1991).

Previous studies have shown that both blood QUIN levels (Heyes and Markey 1988b), as well as tissue (Moroni et al. 1984; Heyes and Markey 1988b) and extracellular (During et al. 1989a, 1989b) brain QUIN levels, rise significantly following increased L-TRP availability. The time course for the brain extracellular QUIN changes displayed in both PCS and control rats showed an early rapid increase following the first loading dose of L-TRP. About 3 hours after the loading dose the QUIN levels reached a peak level at about 20 to 30 times that of the basal levels. The subsequent lower doses $(50 \mathrm{mg} / \mathrm{kg})$ of L-TRP resulted in the achievement of a plateau level of similar degree in both PCS and sham. This phenomenon could indicate either a saturation of the efflux systems carrying QUIN out from the CNS or, perhaps more probable, a saturated QUIN metabolism in the brain. The enzyme responsible for the catabolism of QUIN in the brain is quinolinic acid phosphoribosyltransferase (E.C. 2.4.2.19; QPRT). In the rat brain, the maximal velocity of reaction for QPRT has been demonstrated to be relatively low (Speciale et al. 1987). Hence, it can be speculated that brain QPRT activity may be saturated after excess exposure to QUIN. In this context it should also be pointed out that the enzyme responsible for the major source of kynurenine production in the body is hepatic tryptophan-2,3-dioxygenase (E.C. 1.13.11.11). Several studies have demonstrated that the QUIN found in the brain under normal conditions probably results from a peripheral QUIN production in the liver followed by a passive diffusion (Kitt and Spector 1987) of QUIN in the blood across the blood-brain barrier (BBB) into the brain (Heyes et al. 1993, see also Bergqvist et al. 1995a). In normal conditions (e.g., without brain inflammation), no direct conversion of L-TRP into QUIN has been demonstrated to occur (Heyes et al. 1993). Hence, in rats with experimental hepatic dysfunction (i.e., the PCS rats) it could be anticipated that peripheral QUIN production is lower than in the sham-operated controls, and a possible consequence of this should be that the brain levels of QUIN would be lower after PCS than under normal circumstances. Accordingly, this hypothesis was supported by the findings in both the present investigation and a recent report (Bergqvist et al. 1995a). However, whether a decreased brain QUIN level associated with PCS in the rat is solely due to hepatic dysfunction per se or whether some other factor(s) may also come into play cannot be determined at present. Future investigations on this matter are clearly warranted. It should also be pointed out that the QUIN precursor kynurenine can 
cross the BBB and that enzymes converting kynurenine to QUIN are indeed present in the brain (Heyes et al. 1993). Accordingly, the elevated brain extracellular QUIN levels following elevated L-TRP availability seen in the present study might not only be accounted for by an increased peripheral QUIN production and diffusion over the $\mathrm{BBB}$, but a possibly increased peripheral kynurenine formation with a subsequently increased BBB passage of kynurenine followed by the enzymatic conversion into QUIN also has to be taken into account.

The present study shows that there is no major qualitative difference with regard to QUIN levels between PCS rats and sham-operated controls in the immediate response to a L-TRP load. Levels of QUIN of around $100 \mathrm{nmol} / \mathrm{L}$ have been demonstrated to cause focal degeneration to corticostriatal cell cultures in vitro (Whetsell and Schwarcz 1989). The QUIN values displayed both by the sham and PCS rats following the L-TRP provocation (approximately $200 \mathrm{nmol} / \mathrm{L}$ ) are thus above this level of neurotoxicity. The L-TRP challenge utilized in the current investigation was, however, not aiming at the possible neurotoxic effects of QUIN following the provocation in the short- as well as longterm perspective but, rather, a description of the immediate neurochemical events that could follow such a L-TRP load. Further studies specifically investigating possible neurotoxic effects of QUIN following provocation with L-TRP are thus warranted in the future. Moreover, a more intricate delineation of exogenous systemic L-TRP effects on brain L-TRP and QUIN turnover would necessitate a broader procedure of varying single doses of administered L-TRP and also of repeated dosing of L-TRP given to PCS as well as sham-operated controls. Intravenous systemic versus oral challenges of L-TRP administration also should be undertaken to define aspects such as possible differences between bioavailability in the PCS group and in controls.

In the control rats of the present study, brain extracellular L-TRP concentrations peaked about 1 hour after L-TRP administration, which is consistent with previous reports from a similar challenge (Hutson et al. 1985; Carboni et al. 1989; Carboni and Di Chiara 1989), although in these reports the dosage regimens of L-TRP were slightly different from those in the present study. In the L-TRP-challenged PCS rats in the present study the L-TRP levels reached a peak value within a similar time frame as the L-TRP-challenged sham rats. This value for the PCS rats, however, remained constant throughout the L-TRP challenge experiment. This different time course may be explained by the fact that PCS rats already exhibit elevated brain extracellular L-TRP levels before L-TRP challenge, possibly because of an impaired liver L-TRP metabolism. The ability to metabolize L-TRP has, for example, been demonstrated to be lower in cirrhotic patients (Rössle et al. 1986). Accordingly, following a superimposed L-TRP load to PCS rats, plasma L-TRP levels might significantly increase and the transport of L-TRP from the blood to the CNS might hence be saturated. Although the plasma L-TRP levels are further increased after the L-TRP challenges, the transport of L-TRP into the brain might be kept on a constant level and levels of brain extracellular L-TRP do therefore not increase any further in the L-TRP-challenged PCS rats.

The progressively declining brain extracellular L-TRP levels during the experiments in the saline-administered rats may be due to a drainagelike effect of the probe or, possibly, to normal well-known diurnal variations appearing in the brain for this aromatic amino acid. For example, previous studies have shown that the brain tissue L-TRP content decreases during the light period of the day and increases during the night (Crandall and Fernstrom 1981).

In summary, the present study demonstrates significantly lower basal brain extracellular fluid QUIN levels in chronic PCS rats than in sham-operated controls. Administration of the precursor amino acid L-TRP significantly elevated the QUIN levels in both sham and PCS rats compared to their respective basal QUIN levels. No clear qualitative difference in the QUIN response to this change in L-TRP availability could, however, be seen between sham and PCS rats. We conclude that chronic experimental $\mathrm{HE}$ is not associated with an increased brain extracellular QUIN concentration. These findings, in conjunction with our previous results, do thereby not support a theory of involvement of QUIN in the development of HE.

\section{ACKNOWLEDGMENTS}

The authors are grateful to Kerstin Haraldsson and AnnaKarin Oldén for their skillful technical assistance. We also thank Professor Anders Björklund at the Department of Medical Cell Research, Lund University, Lund, Sweden, for providing space and equipment for the microdialysis experiments.

The generous financial support by the Swedish Society of Medicine, the Ake Wibergs Foundation (Stockholm, Sweden), the Medical Faculty of Lund University, the Crafoordska Foundation, the Anna-Lisa and Sven-Eric Lundgrens Foundation for Medical Research, the Torsten and Elsa Segerfalks Foundation, the Maggie Stephens Foundation, Magnus Bergvalls Foundation, Fredrik and Ingrid Thurings Foundation, and the Royal Physiographic Society (Lund, Sweden) is gratefully acknowledged.

\section{REFERENCES}

Baldessarini RJ, Fischer JE (1979): Serotonin metabolism in rat brain after surgical diversion of the portal venous circulation. Nature New Biol 245:25-27

Basile AS, Saito K, Al-Mardini H, Record CO, Hughes RD, 
Harrison P, Williams R, Li Y, Heyes MP (1995a): The relationship between plasma and brain quinolinic acid levels and the severity of hepatic encephalopathy. Gastroenterology 108:818-823

Basile AS, Saito K, Li Y, Heyes MP (1995b): The relationship between plasma and brain quinolinic acid levels and the severity of hepatic encephalopathy in animal models of fulminant hepatic failure. J Neurochem 64:2607-2614

Bengtsson F (1992): Neurotransmission failure in hepatic encephalopathy involving the combined action of different brain tryptophan-related pathology: A speculative synthesis. In Ishiguro I, Nagatsu T, Nagamura $Y$ (eds), Advances in Tryptophan Research 1992. Toyoake, Japan, Fujita Health University Press, pp 303-308

Bengtsson F, Bugge M, Johansen KH, Butterworth RF (1991): Brain tryptophan hydroxylation in the portacaval shunted rat: A hypothesis for the regulation of serotonin turnover in vivo. J Neurochem 56:1069-1074

Bergeron M, Pomier Layrargues G, Butterworth RF (1989): Aromatic and branched-chain amino acids in autopsied brain tissue from cirrhotic patients with hepatic encephalopathy. Metab Brain Dis 4:169-176

Bergqvist PBF, Heyes MP, Bugge M, Bengtsson F (1995a): Brain quinolinic acid in chronic experimental hepatic encephalopathy: Effects of an exogenous ammonium acetate challenge. J Neurochem 65:2235-2240.

Bergqvist PBF, Vogels BAPM, Bosman DK, Maas MAW, Hjorth S, Chamuleau RAFM, Bengtsson F (1995b): Neocortical dialysate monoamines of rats after acute, subacute, and chronic liver shunt. J Neurochem 64:12381244

Butterworth RF (1992): Evidence that hepatic encephalopathy results from a defect of glutamatergic synaptic regulation. Mol Neuropharmacol 2:229-232

Carboni E, Di Chiara G (1989): Serotonin release estimated by transcortical dialysis in freely-moving rats. Neuroscience 32:637-645

Carboni E, Cadoni C, Tanda GL, Di Chiara G (1989): Calcium-dependent, tetrodotoxin-sensitive stimulation of cortical serotonin release after a tryptophan load. J Neurochem 53:976-978

Cascino A, Cangiano C, Fiaccadori F, Ghinelli F, Merli M, Pelosi G, Riggio O, Rossi-Fanelli F, Sacchini D, Stortoni M, Capocaccia L (1982): Plasma and cerebrospinal fluid amino acid patterns in hepatic encephalopathy. Dig Dis Sci 27:828-832

Crandall EA, Fernstrom JD (1983): Effect of experimental diabetes on the levels of aromatic and branched-chain amino acids in rat blood and brain. Diabetes 32:222-230

Cummings MG, Soeters PB, James JH, Keane JM, Fischer JE (1976): Regional brain indoleamine metabolism following chronic portacaval anastomosis in the rat. J Neurochem 27:501-509

Curzon G, Kantamaneni BD, Fernando JC (1975): Effects of chronic porto-caval anastomosis on brain tryptophan, tyrosine and 5-hydroxytryptamine. J Neurochem 24: 1065-1070

During MJ, Freese A, Heyes MP, Swartz KJ, Markey SP, Roth RH, Martin JB (1989a): Neuroactive metabolites of
L-tryptophan, serotonin and quinolinic acid, in striatal extracellular fluid. Effect of tryptophan loading. FEBS Lett 247:438-444

During MJ, Heyes MP, Freese A, Markey SP, Martin JB, Roth RH (1989b): Quinolinic acid concentrations in striatal extracellular fluid reach potentially neurotoxic levels following systemic L-tryptophan loading. Brain Res 476:384387

Heyes MP, Markey SP (1988a): $\left({ }^{18} \mathrm{O}\right)$ quinolinic acid: Its esterification without back exchange for use as internal standard in the quantification of brain and CSF quinolinic acid. Biomed Environ Mass Spectrom 15:291-293

Heyes MP, Markey SP (1988b): Quantification of quinolinic acid in rat brain, whole blood, and plasma by gas chromatography and negative chemical ionization mass spectrometry: Effects of systemic L-tryptophan administration on brain and blood quinolinic acid concentrations. Anal Biochem 174:349-359

Heyes MP, Saito K, Major EO, Milstien S, Markey SP, Vickers $\mathrm{JH}$ (1993): A mechanism of quinolinic acid formation by brain in inflammatory neurologic disease: Attenuation of synthesis from L-tryptophan by 6-chlorotryptophan and 4-chloro-3-hydroxyanthranilate. Brain 116:1425-1450

Hjorth S, Sharp T (1993): In vivo microdialysis evidence for central serotonin ${ }_{1 \mathrm{~A}}$ and serotonin ${ }_{1 \mathrm{~B}}$ autoreceptor blocking properties of the beta adrenoceptor antagonist (-)penbutolol. J Pharmacol Exp Ther 265:707-712

Hutson DG, Ono J, Dombro RS, Levi JU, Livingstone A, Zeppa R (1979): A longitudinal study of tryptophan involvement in hepatic coma. Am J Surg 137:235-239

Hutson PH, Sarna GS, Kantamaneni BD, Curzon G (1985): Monitoring the effect of a tryptophan load on brain indole metabolism in freely moving rats by simultaneous cerebrospinal fluid sampling and brain dialysis. J Neurochem 44:1266-1273

Jellinger K, Riederer P, Rausch WD, Kothbauer P (1978): Brain monoamines in hepatic encephalopathy and other types of metabolic coma. J Neural Transm 14:103-120

Kalén P, Kokaia M, Lindvall O, Björklund A (1988): Basic characteristics of noradrenaline release in the hippocampus of intact and 6-hydroxydopamine-lesioned rats as studied by in vivo microdialysis. Brain Res 474:374379

Kitt TM, Spector R (1987): Transport of quinolinic acid into rabbit and rat brain. Neurochem Res 12:625-628

Mans AM, Biebuyck JF, Shelly K, Hawkins RA (1982): Regional blood-brain barrier permeability to amino acids after portacaval anastomosis. J Neurochem 38: 705-717

Mans AM, DeJoseph MR, Davis DW, Viña JR, Hawkins RA (1990): Early establishment of cerebral dysfunction after portacaval shunting. Am J Physiol 259:E104-E110

Moroni F, Lombardi G, Carlà V, Moneti G (1984): The excitotoxin quinolinic acid is present and unevenly distributed in the rat brain. Brain Res 295:352-355

Moroni F, Lombardi G, Carlà V, Lal S, Etienne P, Nair NPV (1986a): Increase in the content of quinolinic acid in cerebrospinal fluid and frontal cortex of patients with hepatic failure. J Neurochem 47:1667-1671

Moroni F, Lombardi G, Carlà V, Pellegrini D, Carassale GL, Cortesini C (1986b): Content of quinolinic acid and of other tryptophan metabolites increases in brain regions 
of rats used as experimental models of hepatic encephalopathy. J Neurochem 46:869-874

Ono J, Hutson DG, Dombro RS, Levi JU, Livingstone A, Zeppa R (1978): Tryptophan and hepatic coma. Gastroenterology 74:196-200

Paxinos G, Watson C (1982): The Rat Brain in Stereotaxic Coordinates. Orlando, FL, Academic Press

Rössle M, Luft M, Herz R, Klein B, Lehmann M, Gerok W (1984): Amino acid, ammonia and neurotransmitter concentrations in hepatic encephalopathy: Serial analysis in plasma and cerebrospinal fluid during treatment with an adapted amino acid solution. Klin Wochenschr 62: 867-875

Rössle M, Herz R, Klein B, Gerok W (1986): TryptophanMetabolisms bei Lebererkrankungen: Eine pharmakoki- netische und enzymatische Untersuchung. Klin Wochenschr 64:590-594

Smith AR, Rossi-Fanelli F, Ziparo V, James JH, Perelle BA, Fischer JE (1978): Alterations in plasma and CSF amino acids, amines and metabolites in hepatic coma. Ann Surg 187:343-350

Speciale C, Okuno E, Schwartcz R (1987): Increased quinolinic acid metabolism following neuronal degeneration in the rat hippocampus. Brain Res 436:18-24

Stone TW (1993): Neuropharmacology of quinolinic and kynurenic acids. Pharmacol Rev 45:309-379

Whetsell WO Jr, Schwarcz R (1989): Prolonged exposure to submicromolar concentrations of quinolinic acid causes excitotoxic damage in organotypic cultures of rat corticostriatal system. Neurosci Lett 97:271-275 